\title{
Des femmes et des livres
}

\section{Pascal Dethurens}

\section{(2) OpenEdition}

Journals

Édition électronique

URL : http://journals.openedition.org/rbnu/1200

DOI : $10.4000 /$ rbnu. 1200

ISSN : 2679-6104

\section{Éditeur}

Bibliothèque nationale et universitaire de Strasbourg

\section{Édition imprimée}

Date de publication : 1 novembre 2016

Pagination : 62-71

ISBN : 9782859230630

ISSN : 2109-2761

\section{Référence électronique}

Pascal Dethurens, «Des femmes et des livres », La Revue de la BNU [En ligne], 14 | 2016, mis en ligne le 01 janvier 2020, consulté le 13 décembre 2020. URL : http://journals.openedition.org/rbnu/1200 ;

DOl : https://doi.org/10.4000/rbnu. 1200

\section{(ब) $10(0$}

La Revue de la BNU est mise à disposition selon les termes de la Licence Creative Commons Attribution - Pas d'Utilisation Commerciale - Partage dans les Mêmes Conditions 4.0 International. 


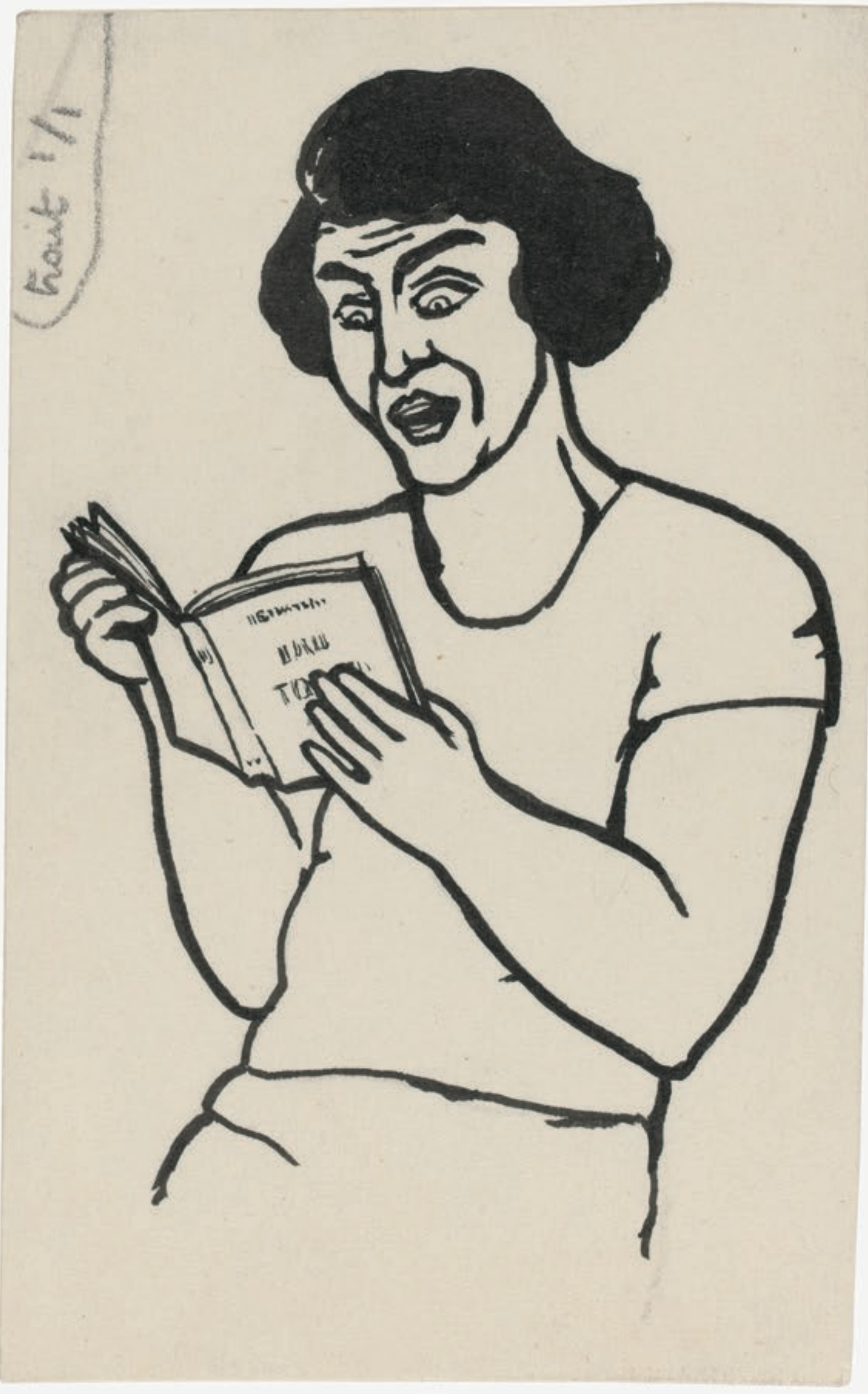




\section{DES FEMMES ET DES LIVRES}

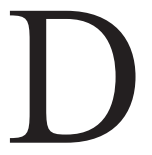

es femmes qui lisent, dans l'art ? On ne les compte plus, elles sont aussi nombreuses que les lectrices des bibliothèques, on aimerait dire. Aussi nombreuses et diverses qu'elles, aussi belles et intrigantes qu'elles parfois, toutes silencieuses, absorbées dans un acte qui les coupe de nous parce qu'il les replie sur leur mystère. Ce qu'elles lisent, comment, pourquoi, on pourrait le savoir si on le leur demandait. Sauf que le règlement prévoit qu'on se taise. Quel dommage. Pensez : toutes ces heures à rester sourdes au monde, à se fermer à nous autrement dit, on se dit que ce temps doit être plus précieux que tout. Mais les lectrices n'ont pour nous aucun regard, elles n'ont pour nous aucun égard. Tant pis. Allons leur demander ce qu'elles font quand elles ne savent pas qu'on les observe. Chez les peintres donc.

Parce que s'il y a un lieu (on va dire un lieu) où les lectrices sont légion, c'est dans la peinture. Un lieu commun même. Il y en a d'autres : des nues, des promeneuses, des endormies, des joueuses. Mais des lectrices, ce n'est pas la peine d'essayer de les compter, chez les peintres vous en trouvez partout. Elles rempliraient une bibliothèque si on les rassemblait. Mais justement. Pas une bibliothèque tout à fait comme les autres. Imaginez-en une où vous pourriez leur parler à toutes. Pas sûr qu'elles vous répondraient. On l'a dit, elles n'ont pas besoin de vous. Ce n'est pas grave : au moins vous découvririez ce qui les retient dans cette immobilité qui vous fascine. C'est déjà ça. Allons voir.
« Des livres, des livres, des livres... De nouveaux livres encore! Qu'est-ce qu'on peut bien trouver là-dedans ?"

Virginia Woolf

\section{Des femmes savantes}

Ce sont bien elles sans doute que l'on rencontre dans les bibliothèques. Ou, quand elles sont chez elles, celles qui nous frappent en premier, parce que sans nous parler elles nous en disent beaucoup. Sur elles, sur leur monde, sur le nôtre peut-être aussi, puisque nous nous approchons d'elles. Regardez pour commencer le portrait de Laura Battiferri (1560) de Bronzino. La jeune femme tient un livre que, curieusement, elle ne lit pas, mais qu'elle fait lire au spectateur. Elle a un secret à nous révéler indirectement, c'est sûr. Ce livre qu'elle vient de lire, à nous d'en faire autant maintenant. Il est l'indice ou, mieux, la signature secrète, le langage codé du tableau, comme s'il en détenait le sens en miniature. Le jeu de miroir est saisissant et ludique à la fois : il s'agit de rien moins que d'une mise en abyme du portrait, dans laquelle Laura vient de lire un sonnet de Pétrarque adressé, comme on sait, à une autre... Laura, figure emblématique de la femme aimée du poète, qui en a immortalisé la beauté dans son Canzoniere.

Pétrarque, auteur de prédilection des savantes Italiennes ? Bien sûr. Il n'y a qu'à rappeler, quelques dizaines d'années plus tard, la Dame au Canzoniere (1628) d'Andrea del Sarto. Elle, au moins, nous regarde en face, elle ne se contente pas de nous désigner la page lue. Mais Bronzino connaît assez la tradition poétique des cercles cultivés de Florence pour savoir la valeur de son étrange profil. Car si le livre explicitement montré au premier 
plan fait sauter aux yeux le texte d'un sonnet qui vante la beauté physique de la femme, il est un autre livre qui apparaît également dans le tableau. Cet autre livre, enfoui dans l'implicite de l'image, n'en est pas caché pour autant, il se donne à lire dans les lignes du profil reconnaissable entre mille du modèle, surtout depuis que Giotto et Botticelli en ont fixé le contour pour l'éternité. Et celui-ci n'est autre que celui, célébrissime, de... Dante lui-même, Dante l'auteur de la Divine Comédie et chantre de l'amour pour Béatrice, la femme à jamais perdue et à jamais aimée.

Ici le livre visible, au service d'un éloge de l'amour sensible ; là, plus secrètement, le livre invisible, consacré à l'exaltation de l'amour spirituel. Les deux langages amoureux, le profane et le sacré, se retrouvent ainsi présents dans le savant portrait. Manière pour la lectrice de faire aimer en elle et le corps et l'esprit. Pourquoi devoir choisir?

\section{Figures de l'absorption}

C'est avec l'évidence d'un fait que la lecture prend corps sous les traits d'une femme, dans la peinture moderne. Non qu'on n'y trouve pas des hommes en train de lire, certes. Des saint Jérôme à perte de vue, des Faust et des don Quichotte à ne plus savoir quoi faire d'eux. Mais ce qu'il y a de remarquable avec la figure de la lectrice, c'est qu'elle ne se rattache pas à un type. Ça n'est pas l'allégorie de ceci ou de cela. Les lectrices sont toutes ce que chacune est. Tant mieux, on y gagne en précision et en humanité. De la sérénité domestique de La Jeune fille lisant (1769) de Fragonard à la coquetterie raffinée de La Marquise de Pompadour (1758) de Boucher, la femme qui lit s'est progressivement constituée en topos du siècle des Lumières et elle parcourt toute la gamme des sentiments si chers à son siècle.

Là, la lectrice dans sa douceur encore enfantine, dans sa plus exquise simplicité : nous sommes, pourquoi pas, dans l'espace de Paul et Virginie. Nulle inquiétude ne vient perturber le bel ordonnancement du monde, nul trouble rompre l'harmonie dorée de la lectrice et du cadre au centre duquel, insoucieuse jusqu'à l'indifférence, elle dit le calme et la permanence. Ici, au contraire, la lectrice dans la sensualité la plus sophistiquée : nous sommes, n'estce pas, chez Diderot, chez Crébillon. Le livre, à n'en pas douter, annonce l'heure et le moment, au moins autant que le désordre de l'alcôve et, comme cette coquine d'horloge qui indique qu'il est temps, il est le plus important de tous les indices libertins du rendez-vous.

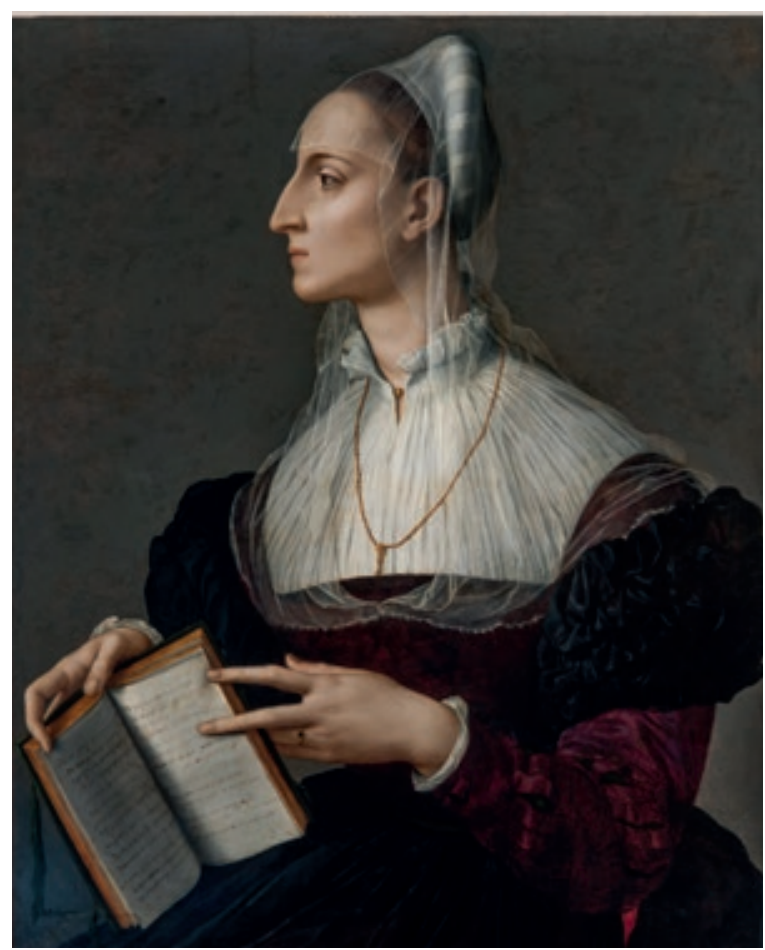

Agnolo Bronzino, Laura Battiferri (1560 ; coll. Palazzo Vecchio, Florence)

Voilà : de la naïveté touchante à l'innocence perdue, de la sérénité silencieuse à l'épanouissement du plaisir, du naturel à l'apprêt, la femme qui lit est peut-être la meilleure messagère, la traductrice la plus fidèle de l'esprit de son temps. Elle est dans tous les cas la femme à la présence fuyante. Elle est là et elle n'est pas complètement là. Ici et ailleurs à la fois. Disparue pour nous, absorbée en elle-même.

On oublie, on oublie beaucoup le monde même chez les lectrices, et c'est le portrait qui fait office d'interprétation, en face du livre lu. Avec ce qu'il se mêle de désir dans toutes ces lectures, d'envie d'évasion mais aussi de plaisir, il suffit de voir à quel point leurs livres les coupent du monde pour comprendre que leur lecture participe chez elles à l'activité même du désir. Quel livre serait exempt de ce besoin ? Si La Liseuse (1874) de Renoir brille, on ne saura jamais pourquoi. Pas de titre indiqué sur la couverture de son livre. A nous d'imaginer, et ce n'est pas un gros effort à faire, que le roman qu'elle tient entre les mains ne la lâchera pas : c'est tellement prenant même qu'elle n'a pas un regard pour le peintre à qui elle 
sert de modèle, pas un pour nous non plus qui sommes ses spectateurs. Non, elle s'absorbe, voilà tout, et tout ce qu'elle demande c'est qu'on ne la dérange pas. Il faut se faire une raison. La lectrice est la femme qui existe sans nous. Sans personne.

Ce qu'on peut préférer à tout avec les figures des femmes surprises en pleine lecture, comme dans La Grande baigneuse au livre (1937) de Picasso, c'est que la lecture est devenue, on aurait envie de dire enfin, l'activité de tous sauf des savants. Une femme lit à la plage, c'est tout. Elle lit, le ciel au-dessus d'elle, la mer juste derrière. À la bonne heure. Et elle oublie le reste, cette femme qui lit. Le reste, à quoi bon ? Extraordinaire pouvoir d'abstraction du monde que le portrait de la lectrice. Dans cette cosmogonie des choses primaires, rien n'importe plus qu'une femme qui ressemble à une statue de géante mise face à son livre. À son livre qui rejoint, du coup, les autres éléments du cosmos, la mer, le ciel, la terre, le soleil. Le savoir n'est de rien ici, il a enfin été dépassé, le souci d'être a enfin été oublié, tout obstacle a été changé en transparence.

Exister ? N'y pensons même pas : demandez à cette lectrice si elle a l'air de s'en préoccuper. Il y a mieux à faire qu'à se poser la question. Voilà peut-être notre plus beau lien à la littérature, dans un après radical de toutes choses, qui est l'allègement que connaissent, seuls, les grands lecteurs.

\section{Au royaume des songes}

Étonnantes, décidément, ces lectrices qu’on rencontre dans la peinture. Tenez, encore, la lectrice de La Tasse de thé (1934) de Derain. Elle vit, on le voit bien, dans l'éloignement que lui procure la solitude de sa lecture, mais aucune impression de tristesse, là non plus, ne se dégage de la scène. Les yeux grands ouverts, cette jeune lectrice ne connaît nulle inquiétude. Tout juste si elle vit. Ce qu'elle ressent ? Pas plus le frisson d'une lecture un peu épicée que le soulagement d'être déchargée des tourments de la passion amoureuse. Ni glacée d'ennui ni ardente de désir. Rien, donc, ou presque rien, à peine si l'on peut mettre des mots là-dessus. Avançons ceci, alors : la lectrice connaît un moment intermédiaire, neutre pourrait-on dire, entre le chaud et le froid, étrangère au moindre trouble, enfin rendue à elle-même.

Songeuse, la femme qui lit, c'est bien le moins qu'on puisse dire. Et elle a de quoi l'être, quand on comprend qu'elle est, en lisant, si proche de l'essentiel, qu'elle affleure l'un des moments cruciaux de sa vie. Bien

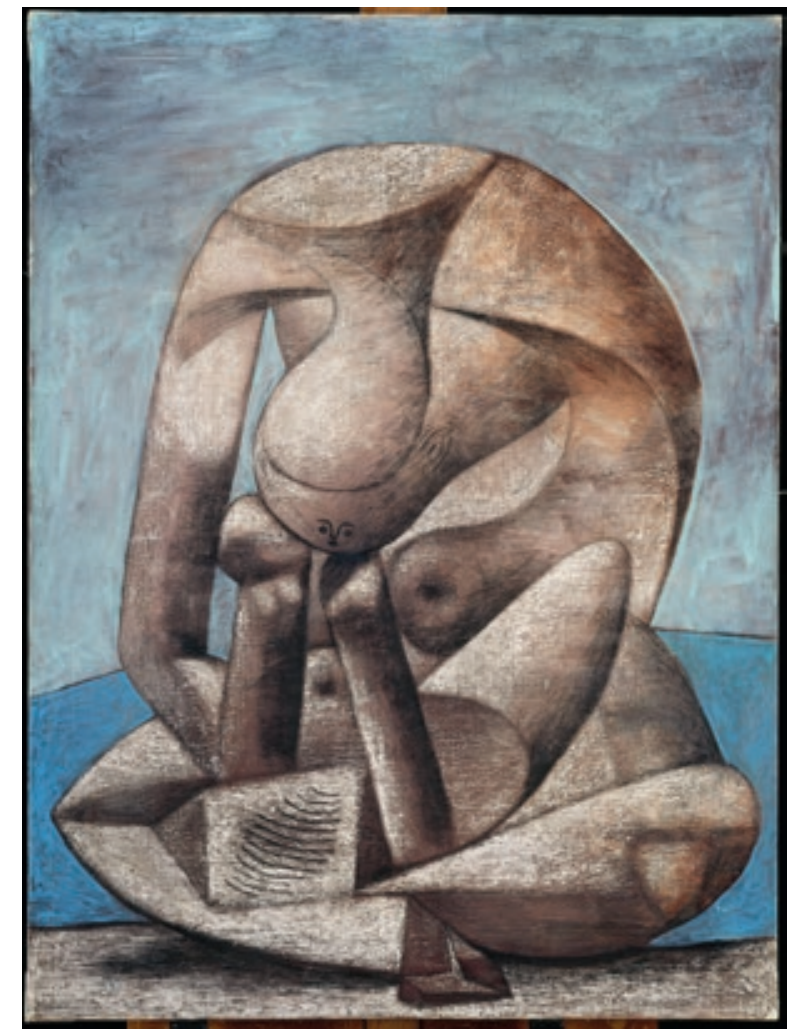

Pablo Picasso, La Grande baigneuse au livre (1937 ; coll. Musée Picasso, Paris)

heureuse encore que sa lecture ne la dérange pas d'ellemême mais, au contraire, qu'elle la restitue à son être le plus profond, à son rêve, on dira. Pour elle, lire ne consiste pas à se faire conduire vers un quelconque ailleurs. Rien de plus faux que d'imaginer que les livres seraient là pour nous distraire. Nous distraire de quoi ? C'est tout l'inverse. Que nous dit d'autre cette femme? Que lire, c'est se lire. La lecture n'a rien à apporter qui lui serait étranger, rien qui lui serait extérieur.

$\mathrm{Au}$ royaume des songes tout nous ramène à nous. Aucun mouvement centrifuge qui, comme le veut l'imagination courante, nous permettrait de nous évader de ce que nous sommes, aucun qui nous inviterait à prendre congé de nous. Mais non. Tout à l'opposé, lire n'enseigne rien qu'on ne sache déjà et sans doute les meilleures lectures sont-elles celles qui nous ramènent, dans un mouvement centripète, au centre de nousmêmes. Ça aussi, les grands lecteurs le savent bien : lire ne les rapproche pas du monde mais les rapproche d'eux. 


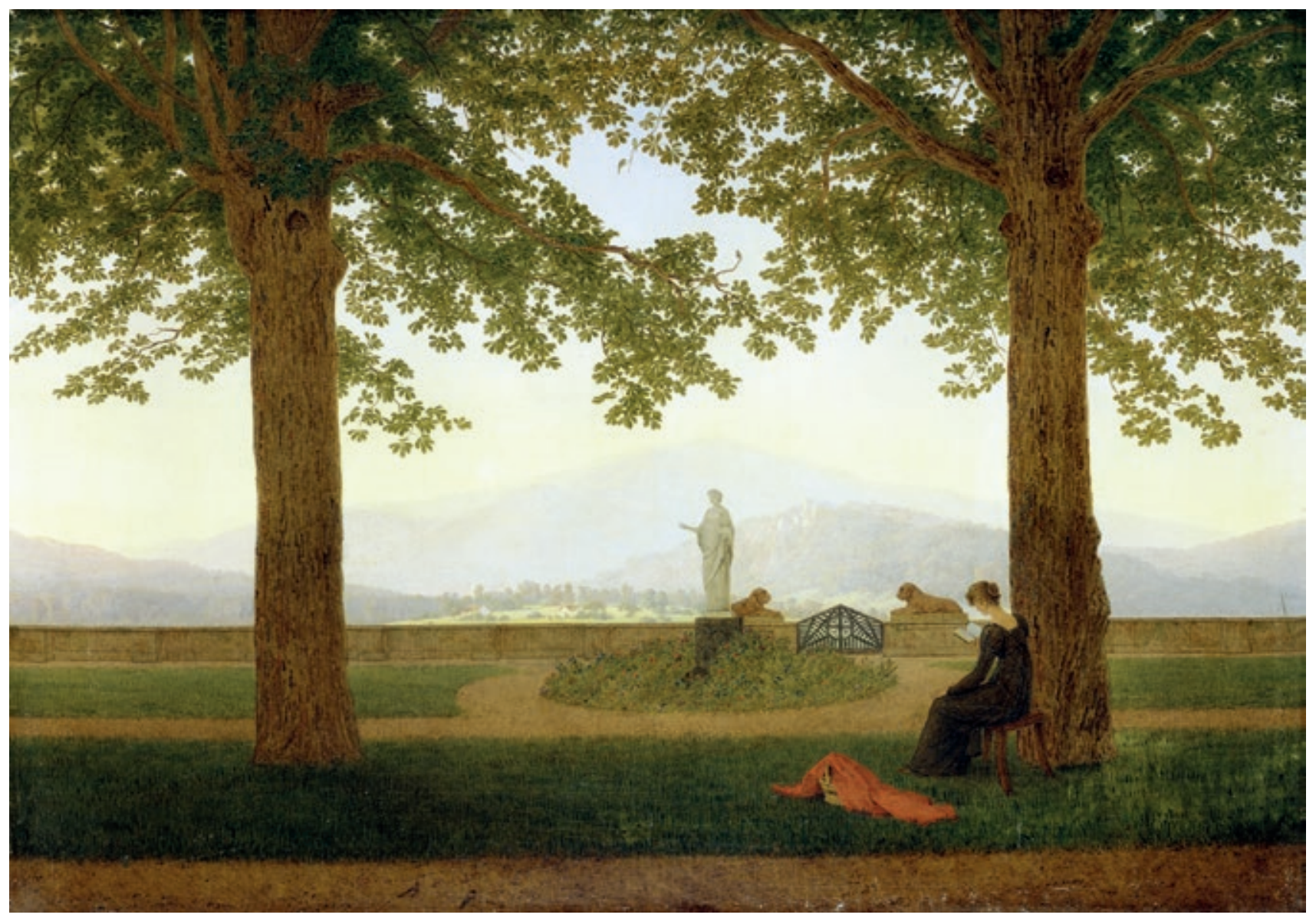

Caspar David Friedrich, La Terrasse du jardin (1811 ; coll. Schloss Charlottenhof, Potsdam)

La lecture, une distraction, une évasion, quoi encore, un amusement, toutes les formes du divertissement ? Il n'a jamais été question de ça. Si ces lectrices sont à la recherche de quelque chose, c'est à la recherche de leurs rêves et, qui sait, de leur interprétation.

Regardez bien comment, dans La Lecture (1927) de Fernand Léger, la femme et l'homme qui lisent, chacun d'eux un livre ouvert à la main, sont sortis de la chaîne des causalités. Ici comme nulle part ailleurs ceux qui lisent oublient le monde, placés dans un hors-champ du social : le livre pourvoit à tout, il tient lieu de monde, et particulièrement quand ce dernier se manifeste avec force. Sourds aux sollicitations du vivant, les lecteursrêveurs ont mieux à faire ailleurs, ils ont la figure de la concentration alanguie, d'une énergie tournée vers l'intérieur. Voilà la fonction du livre dans l'économie du tableau : rappeler le lecteur à son serment, le retenir attaché à lui-même. Le monde peut bien bouger, vibrer, palpiter, comme il le fait toujours dans l'univers de Léger ; le livre sera le point fixe, comme le moyeu autour duquel tout pourra tourner. La lecture, autrement dit, n'a pas la dynamique d'un acte : les figures surprennent au contraire ici par les regards si étonnamment vides, leur indifférente immobilité, leur inexpressivité au centre d'un monde fait toujours de mouvements, de chocs et de stridences.

Mieux : l'ordre de tout arrêter, à quoi la lectrice rêveuse est soumise par son livre, peut aller jusqu'à la changer en statue. Vraiment ? C'est ce qui se passe en tout cas dans La Terrasse du jardin (1811) de C. D. Friedrich. Nul hasard qu'il y ait une statue au milieu exact des lignes horizontales et verticales. Cette statue féminine a tout pour étonner, parce qu'elle déroge à la loi de la structure binaire de la composition (deux arbres, deux 


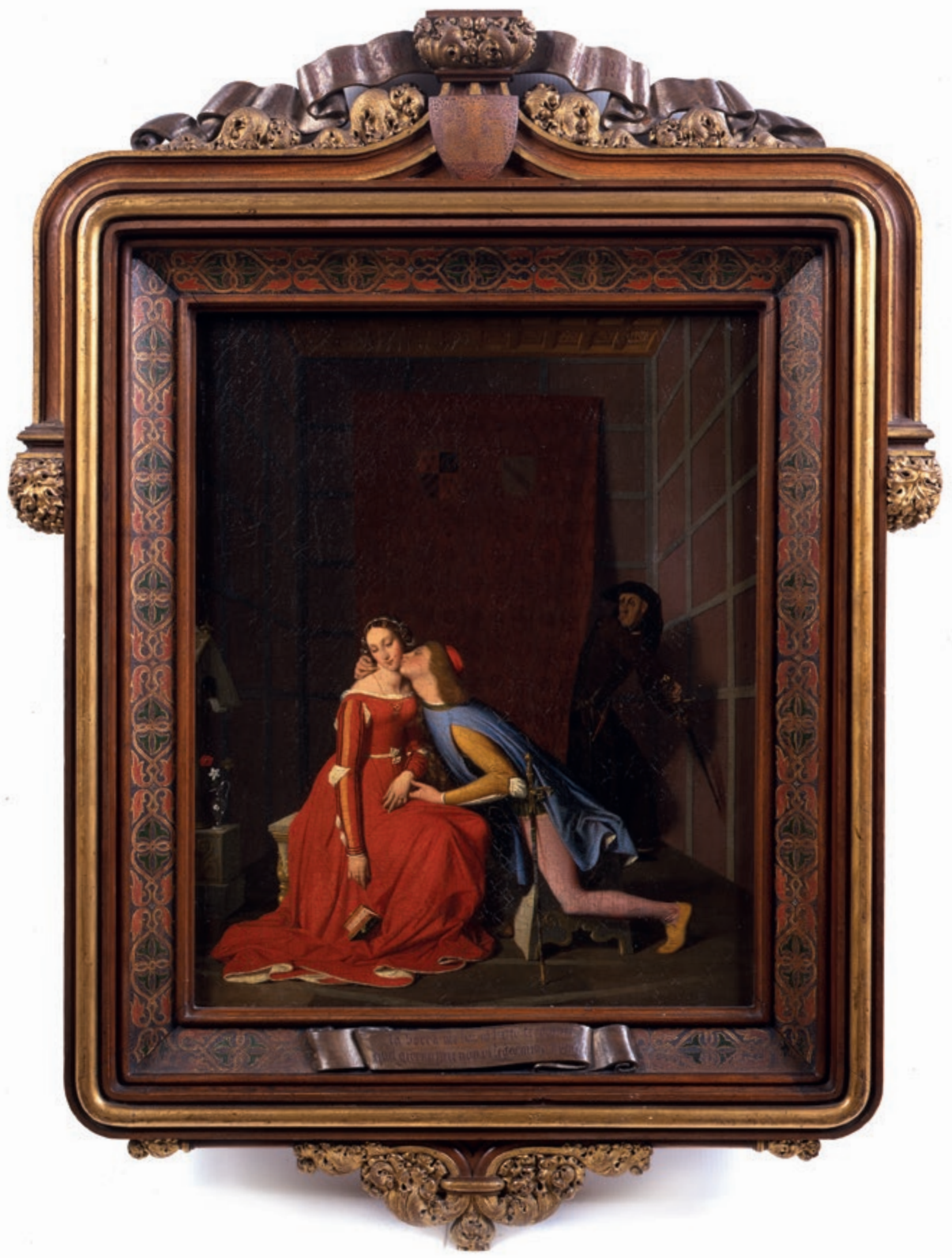

Jean-Auguste-Dominique Ingres, Paolo et Francesca (1819; coll. Musée des Beaux-Arts, Angers) 
lions, deux oiseaux). Mais elle tire sa justification du fait qu'elle symbolise le double, le Doppelgänger de pierre de la femme qui lit et qui, comme la statue, est tournée dans la même direction, les portes du jardin closes, mais le bras tendu vers l'infini, l'infini des songes ouvert pour elle par son livre. Être de pierre, être d'arrêt, la femme qui lit contemple son rêve comme imprimé pour ainsi dire sur les pages de son livre. Toujours fermé, son visage se refuse à trahir le moindre secret sur ce que le livre lui a confié. Mais son silence est éloquent, il prolonge ad libitum le cours de la lecture entamée.

\section{Sidérations}

Et si le livre, au lieu de refermer la lectrice sur elle, au lieu de l'ouvrir aux horizons du rêve, pouvait lui servir à créer un lien amoureux ? Par le pouvoir du livre, elle cesserait alors de vivre dans sa réclusion volontaire. Et il existe justement un couple, un couple réuni autour d'un livre et devenu mythique depuis qu'au chant $\mathrm{V}$ de L'Enfer Dante en évoque l'histoire au début de La Divine comédie (1320). Qu'y a-t-il, pour une femme, à l'origine de l'amour ? Un homme, non. Un homme avec qui lire un livre. Déjà mieux. Accompagné de Virgile, Dante s'arrête un moment dans sa descente aux Enfers, pris d'apitoiement au spectacle de Paolo et Francesca. Comme le veut la légende, Francesca da Rimini, qui a épousé Giovanni Malatesta, s'est éprise de son beau-frère Paolo. Jusque là tout va (presque) bien. Mais le jour arrive où le mari surprend sa femme et son frère en train de lire les aventures de Lancelot (au cours desquelles, on le sait, le chevalier de la Table ronde tombe amoureux de Genièvre, la femme du roi Arthur) et, sans autre forme de procès, les tue. Triste fin pour une si belle lectrice.

On connaît la postérité de ce couple amoureux lié par un livre, son histoire faite de délices et d'horreurs, dans la peinture du siècle romantique. Doré en a fait l'archétype du couple maudit dans l'une de ses illustrations les plus théâtrales de La Divine comédie (1861) où, au mouvement d'abandon des corps lumineux des amants répond la tension de celui du mari, plongé dans la noirceur infernale.

C'est encore la lecture du livre, avec le couple amoureux autour de lui, qui se trouve au point de départ de la composition de Paolo et Francesca (1819) d'Ingres. Suprêmement dramatique, la scène renforce l'innocence amoureuse des protagonistes au premier plan, à la fois par le symbole virginal du vase en cristal et par la

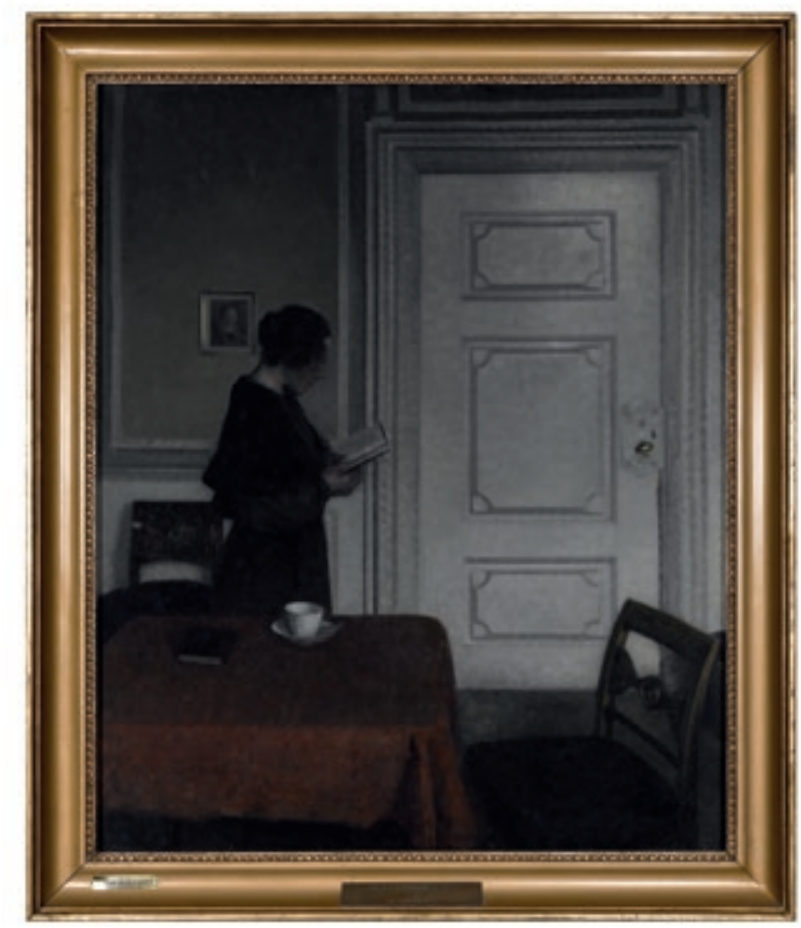

Vilhelm Hammershøi, Femme lisant (1908; coll. Kunstmuseet i Tønder)

position de l'amant transi, dont on croirait de Giotto les couleurs et le visage d'orant séraphique. Il y a dans cet avatar du récit dantesque quelque chose qui tient de la sidération : la lectrice est saisie et son saisissement va causer sa perte.

Curieuse scénographie de la lectrice : son existence est monotone (nous la voyons toujours dans la même situation) et rapide, voire furtive (le temps uniquement d'un portrait), mais elle est prise avec une telle intensité dans la présence qu'elle n'appartient qu'à elle. Si elle semble agir si peu, elle vit en revanche d'une vie intérieure exceptionnelle - captive d'elle-même, fascinée par sa propre histoire.

Occupée d'elle-même au point de ne plus voir ce qui lui arrive, elle vit dans la soumission la plus absolue à son objet, esclave de sa lecture tyrannique. Libérée du monde et de tout, puisqu'elle figure seule sur la toile, la Lectrice soumise (1928) de Magritte ne l'est nullement de 
son livre qui, selon toute apparence, lui fait vivre une expérience inouïe et réclame d'elle la docilité la plus totale. Le titre pour une fois est en relation avec le sujet du tableau, chez le maître belge du surréalisme : alors que le monde est déserté, qu'il est réduit à la minceur d'une paroi bleue contre laquelle la femme terrifiée projette son ombre, rien ne devrait logiquement se produire, et c'est précisément pour cette raison que l'inimaginable a lieu. Paniquée par ce qu'elle lit, la lectrice est prisonnière de son livre comme si ce dernier contenait plus de vie que le monde le plus mouvementé.

\section{Au piège de la solitude}

Captives, donc, prisonnières de leur lecture, façonnées mais plus encore tyrannisées par elle - on pense immanquablement à Emma Bovary -, les femmes qui lisent finissent par disparaître pour faire ce que Blanchot a appelé l'expérience de la "solitude essentielle". Seules : et pourquoi ne les voudrait-on pas telles ? Chez les peintres, elles ne se laissent jamais aussi bien saisir que lorsqu'on les surprend, à l'improviste, comme prises sur le vif, secrètement à l'abri de tout. La tête penchée, le corps replié, elles oublient le monde, c'est entendu, mais elles l'oublient jusqu'à l'oubli de soi, jusqu'au dénuement le plus complet. Pour elles, c'est d'abord le silence et l'éblouissement d'un vertige plus précieux que le bonheur.

Un nouveau monde est créé qui peut alors se lever pour elles toutes, un monde silencieux que peuplent uniquement la méditation solitaire et l'absence à l'Histoire. Énigmatique autant qu'hiératique, inquiétante dans tous les cas, la Femme lisant (1908) de Hammershøi s'est déjà abandonnée au rien, dans une position verticale inattendue qui dramatise l'acte de lecture. Les rares objets épars que l'on voit délaissés, l'immense solitude qui entoure la figure, jusqu'au camaïeu de gris qui dévore l'espace, tout concourt à renforcer autour de la lecture l'effet de néant.

On peut s'étonner du si faible degré de présence chez la femme qui lit. Comme si le livre lu avait la vertu de remplacer ce dont il tient lieu, celle qui s'y abandonne donne son congé au vivant mais, ce faisant, prend le visage austère de la réclusion, mieux, celui, sans mots, de l'être qui a accepté la servitude volontaire. Nulle échappatoire dans une activité quelconque, nul objet par quoi se rattacher au monde. Cette solitude qui est la sienne nous parle de la mélancolie dans ce qu'elle annihile le vouloir-vivre, de cette force innommable qui bloque les énergies.

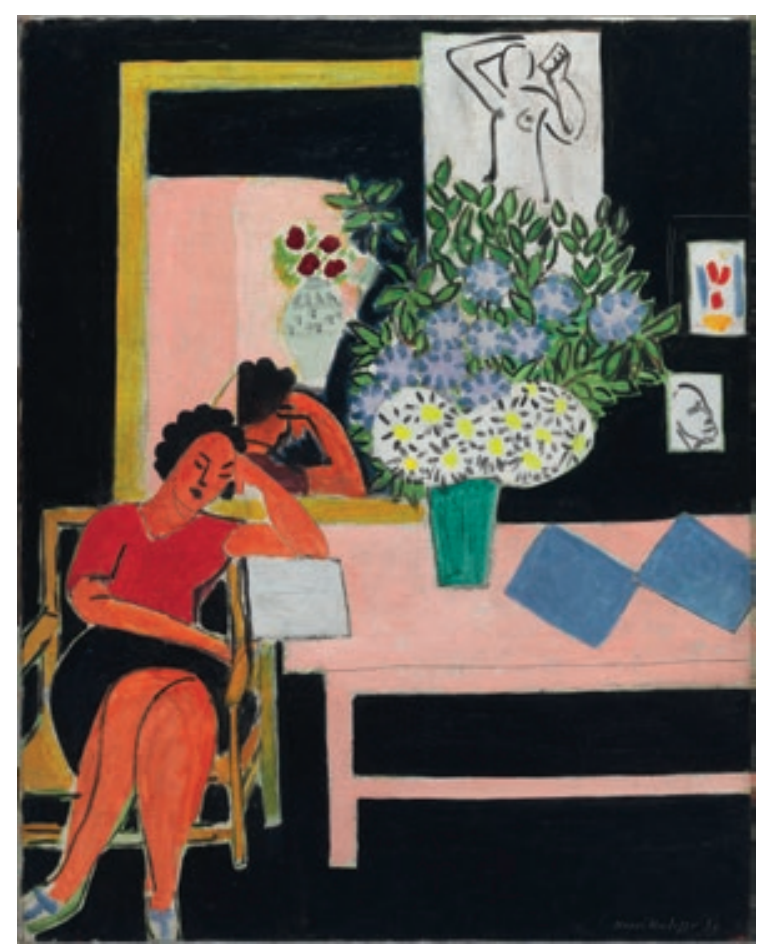

Henri Matisse, Liseuse sur fond noir (1939 ; coll. Musée national d'art moderne)

Échappant elle aussi au monde au point de laisser son intimité s'étaler sous les yeux d'un spectateur devenu voyeur, la lectrice de la Chambre d'hôtel (1931) de Hopper confond, plus que par la froideur qu'elle dégage, par son acquiescement au désespoir, sa résignation au non-être. Rien de plus inhumain que ce mouvement de repli qui est celui de la lectrice scellée sur le silence. À croire que la lecture court indéfiniment vers l'extinction de tout et que ces femmes qui en font l'expérience apprivoisent lentement leur fin. Femmes de l'attente, pas encore de la mort.

\section{Heureuses}

Vraiment ? Lectrices esseulées qu'un halo de glace entoure ou ployées sous le poids de la réflexion, les femmes qui lisent s'abstraient de la voix du monde, dont 
le chaos au moins cesse de leur parvenir. Ce qu'elles offrent, ce que révèlent tous ces visages aussi insensibles à la présence du réel, aussi refermés sur leur propre ravissement, c'est peut-être en dernier ressort qu'au bout de l'expression humaine, à la pointe de la pensée, là où le visage s'absente à tout, il n'y a rien.

Rien : rien à donner, rien à avouer, rien encore à comprendre. Regardez bien une fois encore ces femmes qui lisent, vous verrez chaque fois que leurs yeux se détournent de nous, perdus vers un horizon invisible, les lèvres closes qui semblent vouloir empêcher toute parole. La lectrice exhibe et dérobe, enfouit et révèle en un geste simultané, elle ignore en tout état de cause la logique du partage, parce qu'elle garde tout pour elle. C'est comme ça, il y a des choses qu'on ne prête pas. Il faut l'accepter. Mais si l'envie nous prenait de savoir ce qui les éloigne à ce point de nous, ce qui leur fait avec autant d'évidence préférer ces pages à tout le reste ? Peine perdue. Dédaigneuses de dévoiler même furtivement quelques-unes de ces lignes de si grand prix. Il y a un secret.

Bien malin qui percera le mystère de la femme assise dans La Lecture (1868) de Manet. Son Olympia fait moins la sibylline. Ce n'est pas la même chose, on dira. Mais enfin, que nous dit-elle, à nous fixer comme ça ? Le moins qu'on puisse dire est qu'elle n'a pas l'air décidée à permettre que s'échappe grand-chose d'elle. C'est décevant. On voudrait en savoir plus, rien que pour découvrir ce qui l'a mise dans cet état-là. L'œil égaré dans le vague, le visage tourné pas tout à fait dans notre direction, comme lasse et presque sourde à la voix de l'homme qui lui fait la lecture derrière elle, elle trahit une forme de fatigue qui ne ressemble ni à de la soumission, ni à de la rêverie. Son état ? Plutôt, on aimerait dire, une dilution dans un blanc nébuleux, l'approche imminente d'une extase qui la conduirait à une espèce d'indifférence à tout. Quand de l'autre côté du canapé l'homme concentre son attention sur le texte lu, elle, là sans y être, prostrée, pétrifiée, s'est détournée de toute tension, figure figée dans son propre neutre.

Si on l'imaginait heureuse pourtant ? Le mot de ravissement exprime bien les deux choses à la fois, la plénitude et l'absence. Imaginez un instant connaître ensemble le temps suspendu autour de vous, le monde éloigné loin de vous, la jubilation la plus intense jointe à l'effacement le plus absolu ? Ce qu'en fin de compte réclament les lectrices de plus sérieux, qui sait, il faut voir, ce n'est pas autre chose peut-être que ce qu'on peut attendre secrètement, le droit au bonheur.

Et les lectrices de Matisse, plus que toutes les autres, n'ont rien d'autre à dire que cela, mais alors elles le disent pleinement. Que le bonheur est dans la dissolution du sujet, dans l'habitation du moment, au point qu'il n'existe plus rien d'autre, la lecture tenant lieu de tout. Parler de repli sur soi à propos de ces lectrices serait trop peu : de toute évidence, Marguerite lisant (1906) a la tête penchée et le dos voûté, le visage appuyé sur un coude, en un moment d'oubli absolu de la pose. Elle a mieux à faire qu'à bien se tenir. Mieux à faire qu'à aller à ses occupations. Abîmée comme on l'est quand on tient son affaire. Rien d'extérieur à l'acte de lire n'est là pour encombrer la représentation. Dans un abandon délicieux, la lectrice a oublié le monde et rien ne vient s'imposer du dehors pour s'attacher à la scène. Elle lit, voilà tout, sans rien d'autre à signifier que cela.

S'agit-il encore d'un livre, d'une revue illustrée plutôt, peu importe. La lecture n'est plus mise au service d'une finalité qu'on pourrait appeler la connaissance, elle devient sa propre finalité ou, pour reprendre la formule par laquelle Kant définit l'activité de l'art, elle possède le privilège d'être une " finalité sans fin " - un but en soi.

Cette lecture, pure de tout enseignement, plaisir à l'état pur, on pourrait la croire futile. Comment, notre beau mythe de la lectrice lettrée, savante parfois même, ne connaîtrait plus que cet avatar, un peu léger, d'une femme plongée dans ses magazines ? Souffrons au moins d'admettre que le mythe s'est en effet démocratisé, mais aussi simplifié. Il suffit de rassembler les œuvres de la maturité de Matisse, la Liseuse sur fond noir (1939), la Lectrice à la table jaune (1944), par exemple. Cet univers de fleurs et de couleurs, sur lequel règne un sourire radieux dans la douceur d'un intérieur calme, forme un ensemble de signes qui renvoie à un imaginaire édénique. Eden de pacotille si l'on veut, ou si l'on est grincheux. Mais s'il indiquait ce que l'on pouvait atteindre de meilleur?

Habitante du présent, la lectrice appartient à une humanité qui a enfin trouvé ce à quoi tendent avec plus ou moins de succès ou d'espoir tous les livres et qui est la quiétude, cet état proche de l'introuvable. Ne passons pas trop vite devant les belles lectrices de Matisse : leur bonheur vient d'une intuition vraie, il est en réalité le fruit d'un long apprentissage, au terme duquel le livre n'a plus à se plier à l'austérité de l'étude, ni à s'effacer derrière la recherche de l'absolu. Si cette quiétude est un idéal auquel aspirent secrètement les livres, plus besoin de les lire tous pour arracher du ciel un peu de feu, un seul livre suffit qui les contient et les vaut tous. Les meilleurs livres, comme ceux que nous voyons là, sont ceux qui apportent un peu de savoir, pourquoi pas, mais surtout le plus de saveur possible. 


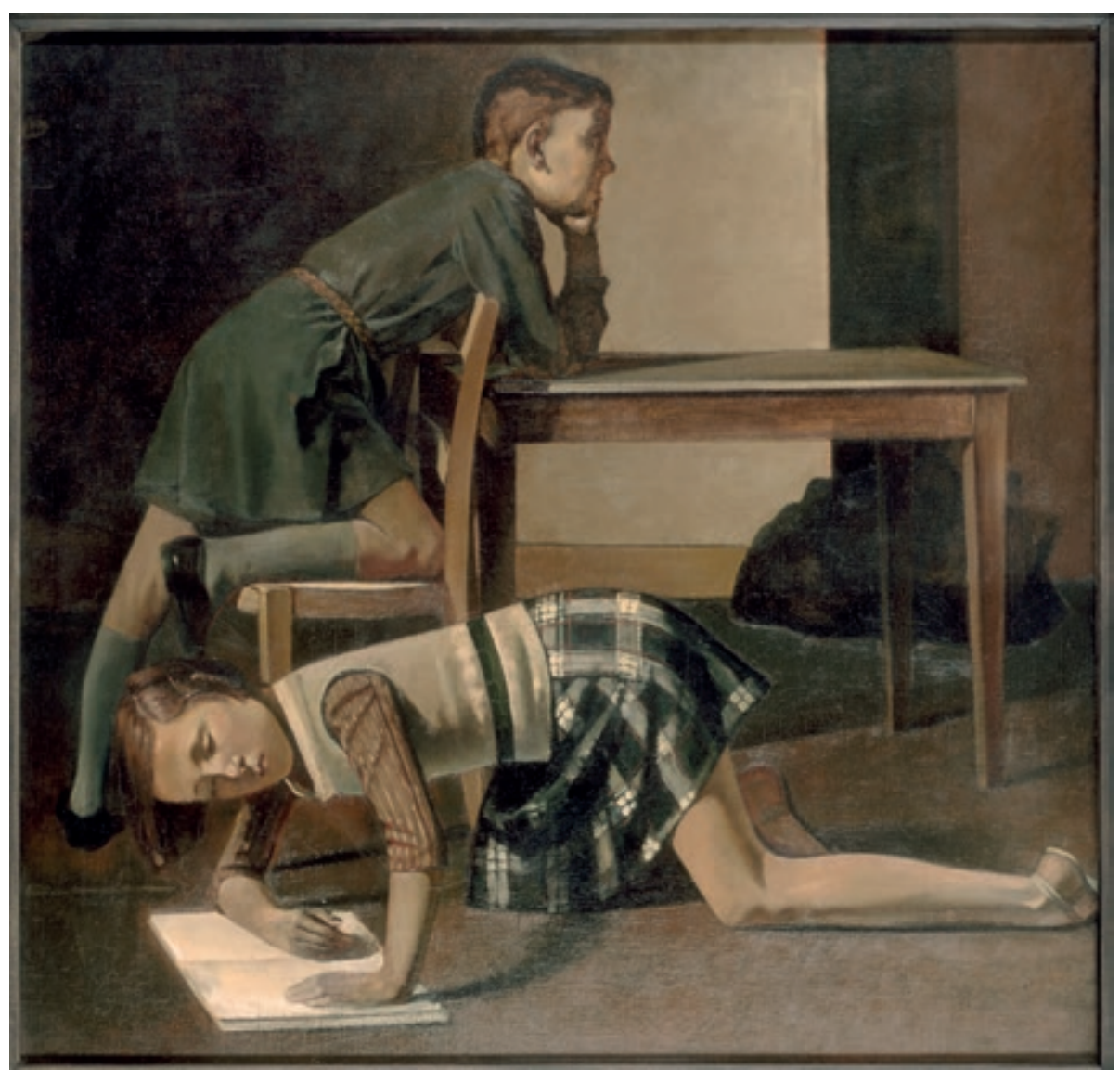

Balthus, Les Enfants

(1937 ; coll. Musée du Louvre)

Qu'y a-t-il, tout compte fait, de plus présent, de plus prégnant sur le souvenir que les lectures d'enfance? Proust a dit la joie - illimitée, ineffable - des retrouvailles avec le monde des lectures enfantines. De ces lectures, il ne subsiste parfois plus même le contenu des histoires, mais seulement le parfum des pages ou la couleur des images. Ces livres-là, il faut les tenir pour ceux qui ont le plus de prix parce que ceux qui les ont lus, quand ils les relisent, se retrouvent tels qu'ils n'ont jamais cessé d'être, des enfants lisant. Balthus a su mieux que personne dire la grâce de ces moments bienheureux de la mémoire, où les livres retiennent à ce point des enfants qu'ils en oublient le regard que porte sur eux le monde extérieur, quand ils ne l'ignorent pas tout bonnement.

Avec cette fille si étrangement agenouillée devant son livre des Enfants (1937) ou du Salon (1942), c'est tout le territoire magique et trouble de l'enfance qui s'étend. Partout la lectrice échappe à la pose officielle, qu'elle congédie pour un état proche du désœuvrement ou voisin du sommeil. Peut-être, comme ces toutes jeunes lectrices, lit-on vraiment lorsqu'on lit pour rien, en marge du portrait de famille toujours, ni complètement assis ni complètement couchés, dans un mode d'être flottant au monde, entre un chat et un piano, en état d'apesanteur pour ainsi dire, comme des passagers clandestins de l'existence.

Curieux bonheur que celui de nos lectrices. Ignorantes de la durée, elles oublient jusqu'à elles-mêmes, enfin délestées du poids d'être en vie, pour être plus présentes ailleurs. Doivent-elles à cette légèreté de paraître comme en lévitation dans plusieurs des tableaux qu'on a vus ? Leur lecture est pleine parce qu'elle est gratuite. Admirable gratuité de ces lectures : elles font concurrence à l'ennui des obligations sociales, elles sont le double éveillé du sommeil. Parce que nos lectrices ont toutes répondu à l'« à quoi bon ? " de la vie et que toutes elles savent qu'il ne sert à rien de vouloir échapper aux plus fortes des emprises. Mais qu'il n'est qu'un seul devoir pour elles, pour nous donc, celui de revenir à soi, pour devenir soi.

\section{Pascal Dethurens}

